\title{
Modulation of the Immune Response by Gangliosides Inhibition of Adherent Monocyte
Accessory Function In Vitro
}

Stephan Ladisch, Lisa Ulsh, Baiba Gillard, and Clifford Wong

Division of Hematology/Oncology and Gwynne Hazen Cherry Memorial Laboratories, Department of Pediatrics, UCLA School of Medicine, Los Angeles, California 90024

bstract. Gangliosides are potent inhibitors of lymphoproliferative responses. Selectively greater inhibitory effects of gangliosides on antigen-induced (vs. mitogen-induced) proliferation have been documented; e.g., $50 \mathrm{nmol}$ of highly purified bovine brain gangliosides $(\mathrm{BBG}) / \mathrm{ml}$ caused $\geq 87 \%$ inhibition of proliferative responses of human peripheral blood mononuclear cells (PBMC) to three soluble specific antigens (Candida, streptokinase-streptodornase, and tetanus toxoid) vs. $\leq 37 \%$ inhibition of responses to three nonspecific mitogens (phytohemagglutinin, concanavalin A, and pokeweed mitogen). The possibility that BBG interfere with adherent monocyte accessory function, upon which responses to soluble specific antigens are strictly dependent, was therefore considered. PBMC were separated into the adherent and nonadherent subpopulations, exposed to $\mathrm{BBG}$, recombined, and their proliferative responses were measured.

Unseparated PBMC preincubated for 48-72 h with $100 \mathrm{nmol} \mathrm{BBG} / \mathrm{ml}$ and then washed to remove unbound BBG exhibited 73-76\% inhibition of subsequent antigeninduced lymphoproliferation. Separate pretreatment of both adherent and nonadherent cell subpopulations in BBG under the same conditions resulted in similar (72-

Portions of this study were reported in abstract form in 1982 Immunobiology. 163:120.

Dr. Ladisch is the recipient of Research Career Development Award 1 KO4 CA 00821 from the National Cancer Institute, and is a Scholar of the Leukemia Society of America.

Received for publication 3 February 1984 and in revised form 30 July 1984.

J. Clin. Invest.

(c) The American Society for Clinical Investigation, Inc. $0021-9738 / 84 / 12 / 2074 / 08 \quad \$ 1.00$

Volume 74, December 1984, 2074-2081
$82 \%$ ) inhibition, which was reproduced by preincubation of only the adherent cells in BBG. Preincubation of only the nonadherent cells in BBG was not inhibitory. Inhibition $(a)$ was independent of whether gangliosides were added in solution or incorporated into liposomes, (b) was abrogated by adding untreated monocytes to cultures containing adherent cells that were preexposed to $\mathrm{BBG}$ (excluding the possibility that BBG was inducing suppression mediated by adherent cells), and (c) was reversible by further incubation of BBG-pretreated adherent cells in control medium. Together, these results delineate a mechanism by which gangliosides modulate lymphoproliferative responses-direct, noncytotoxic, and ultimately reversible inhibition of the accessory function of adherent monocytes.

\section{Introduction}

Gangliosides are sialic acid-containing glycosphingolipids which, in the cell, are localized primarily on the membrane. Recent studies indicate that gangliosides synthesized by proliferating cells may be released into the local environment of the tumor and possibly into the peripheral circulation of tumor-bearing hosts in vivo (1-4). Coupled with the demonstration of potent inhibitory effects of gangliosides on cellular immune responses (4-7), these data stress the importance of understanding the mechanisms of ganglioside-induced inhibition, especially as it may relate to the suppressed cell-mediated immunity associated with certain disease states such as cancer.

To approach this question, we have considered possible explanations for the finding that soluble specific antigeninduced lymphoproliferative responses are quantitatively more susceptible to inhibition by gangliosides than are nonspecific mitogen-induced responses $(4,8)$. Intact monocyte accessory function is an absolute requirement for soluble antigen-specific human lymphoproliferative responses $(9,10)$. The present studies were thus performed to determine which subpopulation 
of human peripheral blood mononuclear cells (PBMC) ${ }^{1}$ is susceptible to inhibition by preincubation with gangliosidesthe adherent cells (predominantly monocytes) or the nonadherent cells (enriched in T lymphocytes). Our results indicate that preincubation with gangliosides selectively paralyzes the accessory functional capability of adherent monocytes in a direct and ultimately reversible manner, but does not impair the subsequent responsiveness of nonadherent cells to antigenic stimulation.

\section{Methods}

Gangliosides. Mixed bovine brain gangliosides (BBG, catalog number 4-6037, lots LA-03608, -9460, -010135, Supelco, Inc., Bellefonte, PA) were further purified by removal of material insoluble in chloroform/ methanol 1:1 and by Sephadex G-50 gel filtration (4). Gangliosides were quantitated as nanomoles lipid-bound sialic acid (LBSA) by the modified resorcinol colormetric assay (11). These extensively purified BBG contained $<0.02 \mathrm{~mol} \%$ protein (Markwell, M. A. K., unpublished results), quantitated as previously described (4), and were free of contaminating phospholipids and neutral glycolipids as assessed by thin-layer chromatography (4).

For addition to the cell cultures, BBG were dissolved in sterile RPMI-1640 (Gibco, Grand Island, NY) and sonicated under $\mathrm{N}_{2}$ for 2 min at room temperature. In selected experiments, BBG were first incorporated into liposomes consisting of cholesterol/lecithin/BBG in a molar ratio of 1:1:1 by previously described procedures (7). Neither BBG alone, cholesterol-lecithin liposomes, nor liposome-incorporated BBG were cytotoxic to PBMC or PBMC subpopulations as assessed by trypan blue dye exclusion at the end of either preincubation or culture periods.

Cells. PBMC were isolated by Ficoll-Hypaque density gradient centrifugation (12) from whole blood collected in preservative-free heparin $(50 \mathrm{U} / \mathrm{ml})$. These cells were washed three times and resuspended in complete medium that consisted of RPMI-1640 supplemented with glutamine $(2 \mathrm{mM})$, penicillin $(50 \mathrm{U} / \mathrm{ml})$, streptomycin $(50 \mu \mathrm{g} / \mathrm{ml})$, and $10 \%$ autologous plasma. PBMC contained $25-30 \%$ monocytes, as assessed by nonspecific esterase staining of cytocentrifuge preparations (13).

Separation of PBMC subpopulations. To obtain the adherent cell subpopulation, $2 \times 10^{5}$ PBMC in $100 \mu$ complete medium were added per well to 96 well flat-bottom microtiter plates (Flow Laboratories, McLean, VA) and incubated for $2 \mathrm{~h}$ at $37^{\circ} \mathrm{C}$ in humidified $95 \%$ air/ $5 \% \mathrm{CO}_{2}$. After three washings to remove nonadherent cells, the wells contained $\sim 5 \times 10^{4}$ adherent cells (>95\% esterase-positive and $>99 \%$ viable by trypan blue dye exclusion). The nonadherent cell subpopulation was isolated by incubating $10^{7}$ PBMC in $5 \mathrm{ml}$ complete medium in $15 \times 100$-mm polystyrene petri dishes for $2 \mathrm{~h}$ at $37^{\circ} \mathrm{C}$ in $95 \%$ air/ $5 \% \mathrm{CO}_{2}$. The dishes were swirled gently and the nonadherent cell population recovered. After a second adherence, the resulting nonadherent cell population contained $<2 \%$ monocytes.

Assessment of lymphoproliferative responses. Proliferative responses

1. Abbreviations used in this paper: BBG, bovine brain gangliosides; Con-A, concanavalin A; PBMC, human peripheral blood mononuclear cells; LBSA, lipid-bound sialic acid; PHA, phytohemagglutinin; PWM, pokeweed mitogen; SKSD, streptokinase-streptodornase. of PBMC were quantitated using published methods (14). Briefly, all cultures contained $2 \times 10^{5}$ cells $/ 0.2 \mathrm{ml}$ complete medium/well. In the experiments in which PBMC subpopulations were recombined, 1.5 $\times 10^{5}$ nonadherent cells were added to wells that contained the adherent cells derived from $2 \times 10^{5}$ PBMC (i.e., $\sim 5 \times 10^{4}$ adherent cells). All conditions, including cell and stimulant concentrations and culture duration were selected to result in optimal ${ }^{3} \mathrm{H}$-thymidine uptake by control PBMC. The duration of culture was $3 \mathrm{~d}$ when the nonspecific mitogens phytohemagglutinin (PHA), concanavalin-A (Con-A), or pokeweed mitogen (PWM) were used as stimulants, and $6 \mathrm{~d}$ when the soluble specific antigens Candida antigen, streptokinase-streptodornase (SKSD), or tetanus toxoid were used. Cultures were then pulsed for $41 / 2 \mathrm{~h}$ with $0.5 \mu \mathrm{Ci}{ }^{3} \mathrm{H}$-thymidine $(6.7 \mathrm{mCi} / \mathrm{mmol})$ and harvested onto glass fiber paper (A. H. Thomas, Philadelphia, PA). ${ }^{3} \mathrm{H}$-Thymidine uptake was quantitated by $\beta$-scintillation counting. Mean net ${ }^{3} \mathrm{H}$ thymidine uptake in triplicate stimulated cultures was determined by subtracting the mean cpm of unstimulated cultures, and percentage inhibition was calculated by comparing mean net ${ }^{3} \mathrm{H}$-thymidine uptake of cultures treated with gangliosides with that of cultures not exposed to gangliosides. The standard error of the mean of triplicate cultures, and of percentage inhibition determined in multiple experiments, was calculated. Statistical significance of the results was determined using the $t$ test.

\section{Results}

Characterization of the sensitivity of mitogen- and antigeninduced lymphoproliferative responses to inhibition by gangliosides. Initial studies were performed to determine the effect of highly purified $B B G$, present during the culture period, on the proliferative responses of human PBMC. In the representative experiment shown in Table I, BBG (50 or $100 \mathrm{nmol} \mathrm{LBSA}$ / $\mathrm{ml}$ culture medium, i.e., 10 or $20 \mathrm{nmol} /$ culture) were added just before the addition of the nonspecific mitogens PHA, Con-A, and PWM, and the soluble specific antigens Candida antigen, SKSD, and tetanus toxoid. At both concentrations of BBG tested in this experiment, the proliferative responses of PBMC to the soluble specific antigens were inhibited to a greater degree than were responses to the nonspecific mitogens. For example, at $50 \mathrm{nmol} \mathrm{LBSA} / \mathrm{ml}$, the greatest inhibition of a mitogen-induced response by BBG added in solution was $37 \%$, while all soluble antigen-specific responses were inhibited by $\geq 87 \%$. The marked and quantitatively similar inhibition by $B B G$ of lymphoproliferative responses to each of the three soluble specific antigens tested characterized BBG as having an effect that was independent of the particular soluble antigen used as the stimulant of lymphoproliferation. When BBG were incorporated into liposomes before addition to the cultures (Table I), slightly higher degrees of inhibition by a given concentration of BBG were observed. The findings demonstrate, however, that the preferential inhibitory effect of BBG on antigen-specific responses was independent of whether BBG were added in solution or in liposomal form.

The selective inhibition of soluble specific antigen-induced proliferative responses is graphically illustrated by the results of the experiment shown in Fig. 1, in which the effects of 
Table I. Sensitivity of Mitogen- and Antigen-induced Human Lymphoproliferative Responses to Inhibition by Gangliosides

\begin{tabular}{|c|c|c|c|c|c|c|}
\hline \multirow[b]{3}{*}{ BBG $(n m o l L B S A / m l)$} & \multicolumn{6}{|l|}{ Stimulant } \\
\hline & \multicolumn{3}{|c|}{ Nonspecific mitogens } & \multicolumn{3}{|c|}{ Soluble specific antigens } \\
\hline & PHA & Con-A & PWM & Candida antigen & SKSD & Tetanus toxoid \\
\hline \multicolumn{7}{|l|}{ In solution } \\
\hline 0 & $62.3 \pm 0.9^{*}$ & $17.8 \pm 1.1$ & $7.0 \pm 0.1$ & $7.5 \pm 2.2$ & $39.9 \pm 0.8$ & $14.6 \pm 4.2$ \\
\hline 50 & $\begin{array}{c}41.6 \pm 2.7 \\
(23) \ddagger\end{array}$ & $\begin{array}{c}11.2 \pm 1.4 \\
(37)\end{array}$ & $\begin{array}{c}6.3 \pm 0.4 \\
(11)\end{array}$ & $\begin{array}{c}1.0 \pm 0.5 \\
(87)\end{array}$ & $\begin{array}{c}3.0 \pm 0.2 \\
(92)\end{array}$ & $\begin{array}{c}0.09 \pm 0.01 \\
(99)\end{array}$ \\
\hline 100 & $\begin{array}{c}32.0 \pm 7.0 \\
(49)\end{array}$ & $\begin{array}{c}6.0 \pm 0.3 \\
(66)\end{array}$ & $\begin{array}{c}7.2 \pm 0.3 \\
(0)\end{array}$ & $\begin{array}{c}0.1 \pm 0.1 \\
(99)\end{array}$ & $\begin{array}{c}0.09 \pm 0.01 \\
(99)\end{array}$ & $\begin{array}{c}0.15 \pm 0.1 \\
(99)\end{array}$ \\
\hline \multicolumn{7}{|c|}{ Liposome incorporated } \\
\hline $0 \S$ & $46.2 \pm 7.2$ & $11.8 \pm 0.5$ & $6.9 \pm 0.2$ & $2.4 \pm 0.4$ & $21.9 \pm 3.4$ & $9.7 \pm 0.6$ \\
\hline 50 & $\begin{array}{c}34.2 \pm 1.7 \\
(26)\end{array}$ & $\begin{array}{c}4.3 \pm 0.4 \\
(64)\end{array}$ & $\begin{array}{c}6.6 \pm 0.1 \\
(4)\end{array}$ & $\begin{array}{c}0.03 \pm 0.03 \\
(99)\end{array}$ & $\begin{array}{c}0.05 \pm 0.01 \\
(99)\end{array}$ & $\begin{array}{c}0.08 \pm 0.01 \\
(99)\end{array}$ \\
\hline $0 \S$ & $54.5 \pm 1.9$ & $12.2 \pm 0.4$ & $6.3 \pm 0.3$ & $2.6 \pm 1.1$ & $21.5 \pm 4.5$ & $5.9 \pm 1.3$ \\
\hline 100 & $\begin{array}{c}15.6 \pm 0.4 \\
(71)\end{array}$ & $\begin{array}{c}0.7 \pm 0.1 \\
(93)\end{array}$ & $\begin{array}{c}5.6 \pm 1.1 \\
(11)\end{array}$ & $\begin{array}{c}0.05 \pm 0.01 \\
(98)\end{array}$ & $\begin{array}{c}0.06 \pm 0.01 \\
(99)\end{array}$ & $\begin{array}{c}0.08 \pm 0.01 \\
(99)\end{array}$ \\
\hline
\end{tabular}
* Mean \pm SEM net $\mathrm{cpm} \times 10^{-3}$ of triplicate cultures. ‡ Parentheses indicate percentage of inhibition vs. net cpm of respective cultures not
containing BBG. $\S$ Liposomes (cholesterol/lecithin, 1:1 molar ratio) not containing BBG. Cholesterol/lecithin/BBG, 1:1:1 molar ratio.

varying concentrations of BBG on lymphoproliferative responses to a nonspecific mitogen, PHA, and to a soluble specific antigen, tetanus toxoid, were compared. At each concentration of BBG tested, inhibition of the response to the specific antigen was significantly greater $(P<0.001)$ than the inhibition of the response to the nonspecific mitogen. This dose-titration experiment further confirms the preferential inhibition of the antigenspecific response by BBG.

The reproducibility of the above findings was confirmed in a series of five separate experiments in which the effect of BBG (100 nmol LBSA/ml) on the proliferative response to the same two stimulants was tested. The mean inhibition of the response to PHA $(32 \pm 2 \%)$ was significantly $(P<0.001)$ less than the mean inhibition of the antigen-induced response $(84 \pm 10 \%)$.

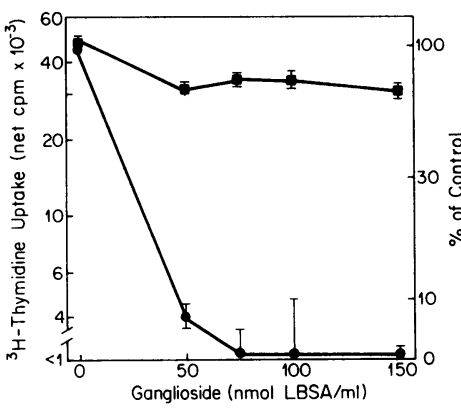

Figure 1. Effect of BBG concentration on proliferative responses of human PBMC. Each point represents the mean $( \pm$ SEM) net ${ }^{3} \mathrm{H}$-thymidine uptake of triplicate cultures containing the indicated concentration of BBG and either PHA (a) or tetanus toxoid (๑).
The marked inhibition of antigen-specific lymphoproliferative responses by BBG was not merely the result of a change in the kinetics of the proliferative response. This is demonstrated by the results shown in Table II, in which the effect of culture duration on the inhibitory effect of BBG was studied. BBG (100 $\mathrm{nmol} \mathrm{LBSA} / \mathrm{ml}$ ) were added just before the stimulating antigen, tetanus toxoid. The cultures were harvested 3-8 d later. Significant inhibition $(P<0.02)$ by BBG was seen at each time point. In fact, while ${ }^{3} \mathrm{H}$-thymidine uptake by control cultures exhibited a 6.5 -fold increase from day 3 to day 6 ,

Table II. Effect of Culture Duration on the Inhibition of Lymphoproliferative Responses by Gangliosides

\begin{tabular}{|c|c|c|c|}
\hline \multirow{2}{*}{$\begin{array}{l}\text { Culture } \\
\text { duration }\end{array}$} & \multicolumn{2}{|l|}{ Treatment } & \multirow{2}{*}{$\begin{array}{l}\text { Inhibition } \\
\text { by BBG }\end{array}$} \\
\hline & Control & BBG & \\
\hline$d$ & & $100 \mathrm{nmol} L B S A / m l$ & $\%$ \\
\hline 3 & $2.3 \pm 0.1^{*}$ & $1.4 \pm 0.2$ & 39 \\
\hline 4 & $3.8 \pm 0.2$ & $2.3 \pm 0.3$ & 39 \\
\hline 5 & $8.0 \pm 0.5$ & $3.3 \pm 0.4$ & 59 \\
\hline 6 & $15.0 \pm 1.9$ & $2.8 \pm 0.1$ & 81 \\
\hline 7 & $16.8 \pm 1.1$ & $2.4 \pm 0.4$ & 86 \\
\hline 8 & $17.7 \pm 2.1$ & $2.3 \pm 0.3$ & 87 \\
\hline
\end{tabular}

* Mean \pm SEM net $\mathrm{cpm} \times 10^{-3}$ of triplicate cultures (stimulating antigen: tetanus toxoid). 
cultures that contained BBG showed very little increase over the entire time period studied. Thus, $>80 \%$ inhibition of ${ }^{3} \mathrm{H}$ thymidine uptake was seen at a time when control cultures exhibited maximal uptake, and maximal ${ }^{3} \mathrm{H}$-thymidine uptake of BBG-containing cultures, observed on day 6 , was only $19 \%$ of that of the control cultures.

Preexposure of PBMC to BBG: effect on subsequent proliferative responses. It has previously been shown that inhibitory effects of gangliosides on PBMC responsiveness could be detected even if unbound gangliosides were removed from the culture medium (by washing of the cell suspension) before the addition of the stimulating mitogen or antigen (7). However, despite rapid binding of ${ }^{3} \mathrm{H}$-labeled gangliosides to PBMC, more prolonged preincubation in gangliosides was necessary to induce this state of unresponsiveness of PBMC (7). We have confirmed this observation to be true of the BBG used in the present studies. In four separate experiments, whole PBMC were preincubated for 24-72 h with BBG (100 nmol LBSA/ml). Then, lymphoproliferative responses to tetanus toxoid of cultures that still contained the gangliosides were compared with those of cultures washed to remove unbound gangliosides (Fig. 2). An increasing degree of inhibition, not abrogated by simple washing of the cells, was observed as the duration of preexposure was lengthened (Fig. 2). Thus, even when followed by washing, 48- or 72-h preincubation of PBMC in BBG resulted in $76 \pm 10$ and $73 \pm 17 \%$ inhibition, respectively, of subsequently tested ${ }^{3} \mathrm{H}$-thymidine uptake, compared to that of cultures that were preincubated in control complete medium $(P=0.025)$. These results indicated that $48-72-\mathrm{h}$ preincubation of separated PBMC subpopulations in BBG should allow determination of which PBMC subpopulation(s) was affected by preincubation in BBG, thereby inducing a state of unresponsiveness of PBMC to subsequent antigenic stimulation.

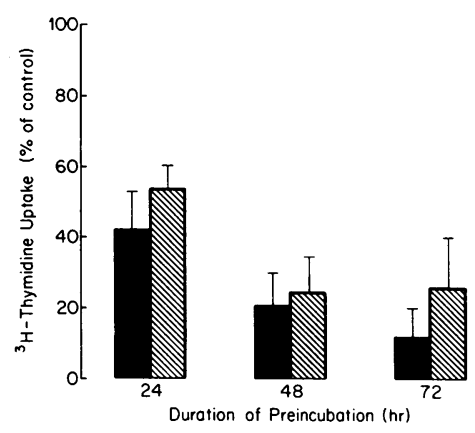

Figure 2. Inhibition of lymphoproliferative responses by preincubation of PBMC with BBG. Normal human PBMC were incubated with BBG (100 nmol LBSA/ml) for 24-72 $\mathrm{h}$ before cultivation with tetanus toxoid. Each bar represents the mean net $( \pm$ SEM $){ }^{3} \mathrm{H}$-thymidine uptake of ganglioside-preincubated PBMC (expressed as percentage of mean net ${ }^{3} \mathrm{H}$-thymidine uptake of PBMC preincubated in control medium) observed in four separate experiments, each performed in triplicate. ${ }^{3} \mathrm{H}$-thymidine uptake by tetanus-stimulated control cultures was $>10^{4} \mathrm{cpm} /$ culture in each experiment. Solid bars: BBG present during preincubation and lymphoproliferation assay periods. Hatched bars: unbound BBG removed after preincubation period, by washing of PBMC, before assessment of lymphoproliferation.
Effect of preexposure of PBMC subpopulations to BBG. To determine whether inhibition of subsequent lymphoproliferative responses resulting from preincubation of PBMC in BBG reflected an effect of BBG on the nonadherent (T lymphocyteenriched) subpopulation, or on the adherent (monocyte-enriched) subpopulation, PBMC were separated into these subpopulations. In two experiments (Fig. 3), the effectiveness of the cell separation procedures and the proliferative response of these two subpopulations when combined after separate preincubation for $72 \mathrm{~h}$ in control medium were tested. Neither subpopulation alone yielded $>1 \%$ of the proliferative response of unseparated cells $(P<0.001)$. In contrast, the recombined subpopulations exhibited a response not significantly different from that of the unseparated PBMC $(P>0.9)$.

Having confirmed the effectiveness of the separation procedure and the functional integrity of the recombined subpopulations, we next studied the effect of separate preincubation of the adherent and nonadherent subpopulations in BBG (100 nmol LBSA/ml). Each subpopulation was preincubated in BBG or in control medium for 4-72 $\mathrm{h}$ and then washed. The subpopulations were then recombined and the proliferative response to a soluble specific antigen (SKSD or tetanus toxoid) measured. The findings of these five experiments (Fig. 4) were: (a) As expected from the results shown in Fig. 2, 48- or 72-h preincubation of both subpopulations in BBG $(a)$ resulted in 82 and 72\% inhibition of the proliferative response, respectively, as compared with preincubation of the subpopulations in control medium $(P<0.01)$. Shorter preincubation did not result in significant inhibition, although a trend towards increasing inhibition was seen after $24 \mathrm{~h}$ of preincubation in BBG. (b) Preincubation of only the adherent cell subpopulation $(\bullet)$ in BBG resulted in inhibition that was not significantly different $(P>0.3)$ from that caused by preincubation of both subpopulations in BBG ( $(\square)$ at any time point. (c) Preincubation of only the nonadherent cell subpopulations in BBG (O) did not result in significant inhibition $(P>0.1)$ at any time point. Taken together, these experimental results suggest that inhibition of lymphoproliferative responses caused by preexposure

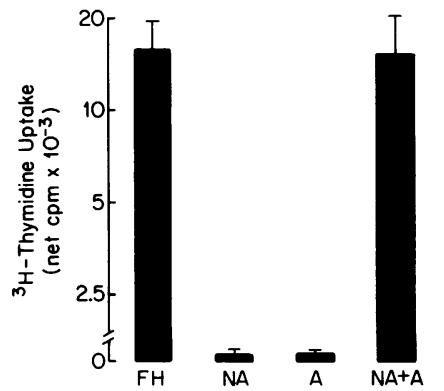

Figure 3. Proliferative responses of PBMC subpopulations. Human PBMC (FH), adherent (A), and nonadherent (NA) subpopulations of PBMC were incubated in control complete medium for $72 \mathrm{~h}$, and then washed. The washed subpopulations were then either cultured separately or recombined, and their proliferative responses to SKSD deter-

mined. Each bar represents the mean net $( \pm S E M){ }^{3} \mathrm{H}$-thymidine uptake that was observed in two experiments, each performed in triplicate. 


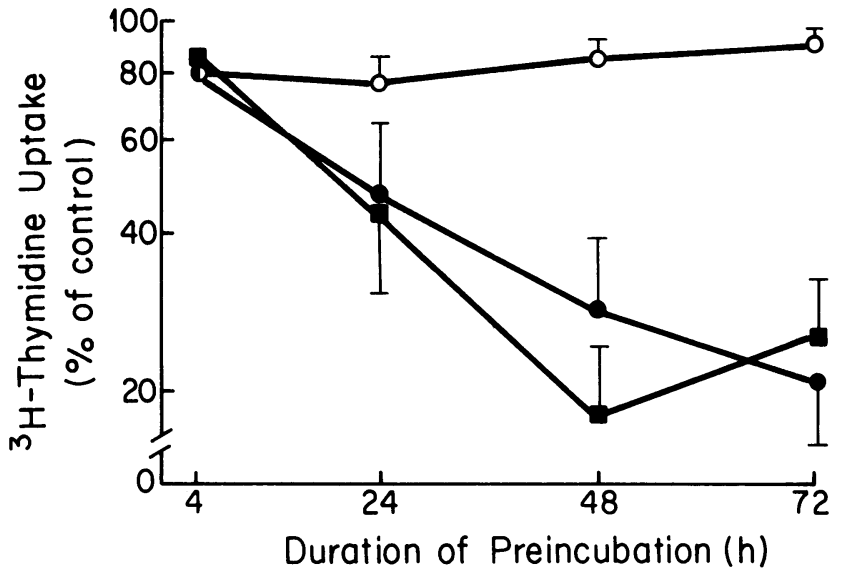

Figure 4. Effects of duration of preincubation of PBMC subpopulations in BBG. Adherent and nonadherent cells were incubated for 4$72 \mathrm{~h}$ in BBG (100 nmol LBSA/ml) (G) or control medium (C), washed, recombined, and lymphoproliferative responses to SKSD or tetanus toxoid assessed. Points represent the mean $( \pm S E M)$ percent net ${ }^{3} \mathrm{H}$-thymidine uptake (as compared with control $\left(\mathrm{A}_{C}+\mathrm{NA}_{C}\right)$ proliferative responses) observed in triplicate cultures of up to five separate experiments at each time point. Control responses $\left(A_{C}+\right.$ $\mathrm{NA}_{C}$ ) to these stimulants were $5-12 \times 10^{3} \mathrm{cpm} /$ culture. Key: $\mathrm{O}, \mathrm{A}_{C}$ $+N A_{G} ; \bullet, A_{G}+N A_{C} ; n, A_{G}+N A_{G}$.

of PBMC to BBG is the result of an effect on the adherent monocyte (and its functional capability as an accessory cell), and not the result of interference with the ability of the nonadherent cell subpopulation to proliferate in response to effective antigenic stimulation.

The delineation of a selective effect of BBG on adherent monocyte accessory function raises several additional questions that were addressed experimentally. These questions are: Is the inhibitory effect the result of toxic effect on the adherent cell subpopulation? Can the inhibitory effect be explained by residual ganglioside adsorbed to the plastic wells and not removed during washing of the adherent cell subpopulation? Does inhibition depend upon the mode of presentation (i.e., in solution or liposome incorporated) of gangliosides to the adherent cell subpopulation?

Previous studies have consistently demonstrated that purified gangliosides are not cytotoxic to $P B M C(4,6-8)$. In the present studies, BBG (100 nmol LBSA/ml) present either during the 72-h preincubation period or during the 6-d culture period were not cytotoxic, as measured by difference in trypan blue dye exclusion between control and BBG-exposed PBMC. Separate preincubation of the adherent and nonadherent PBMC subpopulations in BBG for $72 \mathrm{~h}$ did not reduce their viability $(>90 \%)$. Finally, the possibility that preincubation of the adherent cell subpopulation in BBG could be causing a loss (e.g., by detachment) of cells necessary for intact accessory function was tested. Adherent cells were plated in prescored wells, incubated in either BBG or control medium for $72 \mathrm{~h}$, and washed as in preparation for the lymphoproliferation assay. Then, viable adherent cells in a defined area of the surface of each well were counted. In nine sets of cultures, the mean number of viable cells that remained in BBG-exposed wells was $93 \pm 6 \%$ of the number that remained in the control wells $(P>0.4)$. Therefore, there is no evidence suggesting that a direct cytotoxic effect or loss of adherent monocytes could explain the BBG-induced inhibition of adherent monocyte accessory function.

Since gangliosides adhere to plastic surfaces (15), another possible explanation for inhibition could have been that adsorption of BBG to the plastic wells in which the adherent cells were precultured could have resulted in inhibition of the washed cells, which were subsequently cultured in the same wells to assess lymphoproliferation. This would be highly unlikely, since, at the ganglioside concentration used, $<1 \%$ of the BBG (or $1 \mathrm{nmol} \mathrm{LBSA} / \mathrm{ml}$ ) would be expected to adhere to the plastic (15). We nevertheless tested whether functionally active quantities of BBG remained adsorbed to the plastic surface by adding control PBMC to wells in which gangliosides (100 $\mathrm{nmol} \mathrm{LBSA} / \mathrm{ml}$ complete medium) had been allowed to adsorb for $72 \mathrm{~h}$, after which the wells were rinsed. There was no inhibition of the proliferative response to SKSD of PBMC cultured in BBG-pretreated wells, compared to control wells (30.6 \pm 3.9 vs. $27.2 \pm 1.2 \times 10^{3} \mathrm{cpm}$, respectively). In contrast, the original concentration of BBG (100 nmol LBSA/ml) caused 78\% inhibition of control PBMC responses (6.0 0.6 $\times 10^{3} \mathrm{cpm} /$ culture). Thus, adsorbed BBG were not responsible for the inhibitory effects that resulted from the preincubation of the adherent cell subpopulation in BBG.

To determine whether mode of presentation of BBG (i.e., free or liposome incorporated) might be a factor in causing selective inhibition of adherent monocyte accessory function, BBG were incorporated in cholesterol/lecithin liposomes (as described in Methods). Preincubation of adherent monocytes in liposome-incorporated BBG (50 or $100 \mathrm{nmol} \mathrm{LBSA} / \mathrm{ml}$ complete medium) for $72 \mathrm{~h}$ resulted in 95 and $99 \%$ inhibition, respectively, of the subsequent proliferative responses of control nonadherent cells to tetanus toxoid, compared with the response observed when adherent cells were preincubated in either control complete medium or complete medium containing cholesterol/lecithin liposomes $(P<0.001)$. Thus, both BBG added in solution and BBG incorporated into liposomes markedly inhibit adherent monocyte accessory function.

Reversibility of BBG-induced inhibition of adherent accessory cell function. To determine whether the selective effect (i.e., on the adherent cell subpopulation) of preincubation of PBMC in BBG was the result of induction of suppressor activity in this subpopulation or the result of direct interference of BBG with the accessory functional capability of the adherent monocyte, we tested the ability of untreated monocytes to overcome the inhibitory effect caused by the preincubation of the adherent cell subpopulation in BBG. In this experiment, adherent cells were preincubated with BBG (100 nmol LBSA/ $\mathrm{ml}$ ) for $72 \mathrm{~h}$, washed, and then combined with either control nonadherent cells or control PBMC (the latter containing both 
control adherent and control nonadherent subpopulations). Fig. 5 shows that the addition of control total PBMC to BBGpretreated adherent cells completely abrogated the significant inhibition $(P<0.001)$ observed when only control nonadherent cells were added to BBG-pretreated adherent cells. This effect of the addition of untreated monocytes strongly suggests that BBG directly inhibit the accessory function of adherent monocytes rather than induce suppressor activity in the adherent cell subpopulation.

It was therefore of interest to determine whether the noncytotoxic, direct, inhibitory effect of BBG represented a permanent (i.e., irreversible) effect on adherent monocyte accessory function, or whether prolonged further incubation of BBG-pretreated adherent cells in control medium could restore their functional capability. To test these possibilities, adherent cells were exposed to BBG (100 nmol LBSA/ml) for $72 \mathrm{~h}$ and then washed. The BBG-pretreated adherent cells were then either immediately cultured with nonadherent cells and tetanus toxoid, or incubated for an additional $72 \mathrm{~h}$ in control medium, washed again, and then cultured with nonadherent cells and tetanus toxoid. To eliminate possible remaining monocytes that were not removed by the adherence procedure, the nonadherent cell subpopulation was further purified by E-rosetting and filtration through a Sephadex G10 column to yield a $T$ cell population that was virtually devoid of contaminating monocytes (16). As shown in Fig. 6 (solid bars), preincubation of adherent cells in control medium for 72-144 $\mathrm{h}$ resulted in ${ }^{3} \mathrm{H}$-thymidine uptake of $6-9 \times 10^{3}$ cpm/culture, when control $\mathrm{T}$ cells were added at the end of these preincubation times. Both 72- and 144-h preincubation of adherent cells in BBG, followed by washing and the immediate addition of control $\mathrm{T}$ cells, resulted in $>90 \%$ inhibition of the subsequent lymphoproliferative responses. However, when BBG-pretreated, washed, adherent cells were incubated in control medium for an additional $72 \mathrm{~h}$ before testing their accessory function in the lymphoproliferation assay, a degree of ${ }^{3} \mathrm{H}$-thymidine uptake not significantly different $(P>0.05)$ from that of the respective control cultures was

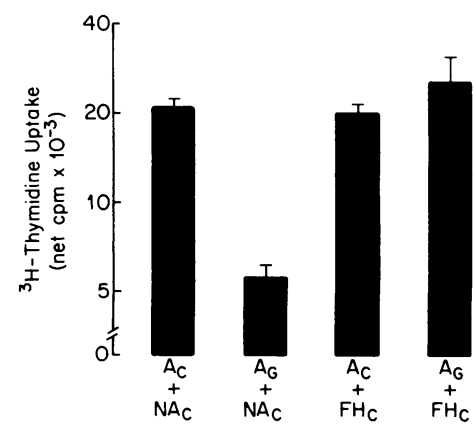

Figure 5. Inhibition of proliferative responses caused by preincubation of adherent cells in BBG: effect of addition of untreated adherent cells. Adherent cells (A) were preincubated in control medium $(C)$ or BBG (G), 100 nmol LBSA/ $\mathrm{ml}$, for $72 \mathrm{~h}$, and then washed. Control nonadherent cells $\left(\mathrm{NA}_{\mathrm{c}}, 1.5 \times 10^{5}\right.$ cells) or $2 \times 10^{5}$ control

PBMC (i.e., nonadherent plus adherent, $\mathrm{FH}_{\mathrm{c}}$ ) were added as shown. Bars represent the mean $\left( \pm\right.$ SEM) net ${ }^{3} \mathrm{H}$-thymidine uptake of triplicate cultures; stimulant: SKSD.

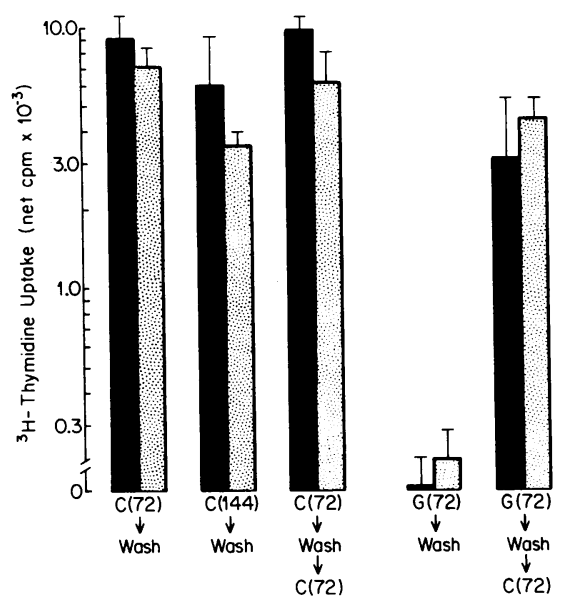

Figure 6. Functional recovery of BBG-pretreated cells after further preincubation in control medium. Adherent cells were incubated in control medium (C) or BBG (100 nmol LBSA/ml) (G) for the time indicated in brackets, washed, combined with control purified $\mathrm{T}$ cells, and the mean net ${ }^{3} \mathrm{H}$-thymidine uptake of triplicate cultures stimulated by tetanus toxoid quantitated. Solid bars: adherent cells preincubated in complete medium (C) or BBG (100 nmol LBSA/ml) $(\mathrm{G})$; stippled bars: adherent cells preincubated in liposomes (cholesterol/ lecithin 1:1) (C) or liposomes containing BBG (100 nmol LBSA/ml) (G); stimulant: tetanus toxoid. Proliferative responses of cultures containing adherent cells preincubated for $144 \mathrm{~h}$ instead of $72 \mathrm{~h}$ in BBG before washing were similar, i.e., $<0.2 \times 10^{3}$ net $\mathrm{cpm} /$ culture.

observed, which demonstrated that inhibition of the accessory functional capability of adherent monotypes by BBG is ultimately reversible. These findings also provide supporting evidence for the previously stated conclusion that exposure of adherent monocytes to BBG did not result in the actual loss (e.g., by detachment) of certain monocytes that were critical for the accessory function. Finally, the addition of BBG incorporated into liposomes (Fig. 6, stippled bars) gave statistically similar results $(P>0.1)$ to those that were obtained when BBG were added in solution.

Taken together, the findings of these studies support the conclusion that BBG directly interfere with the accessory functional capability of adherent monocytes, and do so in a noncytotoxic and reversible manner.

\section{Discussion}

Gangliosides are potent inhibitors of the proliferative responses of PBMC to antigenic stimulation in vitro (4-8). Accumulating evidence of abnormalities in circulating gangliosides in certain disease states associated with immunodeficiency, such as cancer $(1,17,18)$, suggests that inhibition of lymphoproliferation by gangliosides may be of in vivo relevance. This possibility is strengthened by recent findings that the effector cell generated by an intact lymphoproliferative response, the cytotoxic $\mathrm{T}$ cell, is capable of eliminating tumor cells in vivo $(19,20)$. These 
findings underscore the importance of studying immunosuppressive factors, such as gangliosides, which occur in vivo and are present in the local environment (e.g., of tumor) where they might affect immune responses, and of elucidating the mechanisms by which they inhibit lymphoproliferative responses.

Previous studies identified two characteristics of inhibition of lymphoproliferative responses by gangliosides. First, gangliosides present during the culture period directly interfere with the proliferation of mitogen-activated lymphocytes (6), possibly by inhibiting lymphocyte $\mathrm{Ca}++$ influx normally associated with lymphocyte activation (21). Second, preincubation in gangliosides impairs the responsiveness of human PBMC to subsequent activation by stimulants of lymphoproliferation (7). The present studies were addressed to the identification of the immunologic mechanism resulting in this state of impaired responsiveness. This question was approached by determining which of the subpopulation(s) of PBMC was affected by preincubation with gangliosides.

Our results demonstrate that BBG inhibit accessory function of the adherent (monocyte-enriched) subpopulation, which is critical to antigen-induced lymphoproliferative responses. The inhibition is mediated by a direct effect on the functional capability of these cells rather than by the induction of suppressor activity. Also, this state of temporary unresponsiveness, or paralysis, of adherent monocyte accessory function induced by BBG was independent of the mode of presentation of BBG. Although it has previously been suggested that gangliosides added in solution may interact differently with PBMC than do gangliosides incorporated in liposomes (7), our findings indicate that, aside from slight quantitative differences in inhibition associated with the mode of presentation, the mode of presentation does not in itself alter the immunologic mechanism by which these gangliosides act when preincubated with PBMC - that is, abrogation of adherent monocyte accessory function.

Why prolonged preincubation with gangliosides is required to render adherent monocytes nonfunctional with respect to their critical accessory role in the initiation of proliferative responses of PBMC to soluble specific antigens is not yet known. The results suggest, however, that more than just binding (i.e., possibly internalization and/or metabolism) of gangliosides may be required to abrogate this function. Which aspect of accessory function is affected also remains to be determined, but the possibilities include antigen uptake or processing, effective interleukin-1 production and/or release, and effective cellular collaboration of adherent monocytes with the responding lymphocyte subpopulation of PBMC.

The present findings and previous reports identify two PBMC subpopulations that are essential to normal lymphoproliferative responses, adherent monocytes (accessory function) and nonadherent lymphocytes (the proliferating cells), to be affected by gangliosides. Effects on these two PBMC subpopulations are not mutually exclusive. In fact, taken together they provide one explanation for the high degree of inhibition of soluble specific antigen-induced (vs. nonspecific mitogeninduced) lymphoproliferative responses by gangliosides. The initiation of specific antigen-induced lymphoproliferative responses is critically dependent upon intact adherent monocyte accessory function $(9,10)$. This knowledge would predict that modulation by gangliosides of the function of both adherent monocytes (present study) and of lymphocytes (6) might result in very substantial inhibition of antigen-specific responses, as we in fact observed. Since specific antigens are the relevant stimulants of the cellular immune response in vivo, the marked inhibition of antigen-specific lymphoproliferative responses by gangliosides in vitro suggests that these glycolipids may be very potent, biologically relevant, immunoregulatory molecules in vivo.

\section{Acknowledgments}

We thank Dr. Mary Ann K. Markwell for the determination of protein in the lipid samples, Julie Nguyen for assistance, and Dorothy Ross for preparation of the manuscript.

This work was supported by grants CA27701 and HD18171 from the National Institutes of Health, a National Institutes of Health Biomedical Research Support grant, and a grant from the California Institute for Cancer Research.

\section{References}

1. Kloppel, T. M., T. W. Keenan, M. J. Freeman, and D. J. Morré. 1977. Glycolipid-bound sialic acid in serum: increased levels in mice and humans bearing mammary carcinomas. Proc. Natl. Acad. Sci. USA. 74:3011-3013.

2. Koprowski, H., M. Herlyn, Z. Steplewski, and H. F. Sears. 1981. Specific antigen in serum of patients with colon carcinoma. Science (Wash. DC). 212:53-55.

3. Portoukalian, J., G. Zwingelstein, N. Abdul-Malak, and J. Doré. 1978. Alteration of gangliosides in plasma and red cells of humans bearing melanoma tumors. Biochem. Biophys. Res. Commun. 85:916920.

4. Ladisch, S., B. Gillard, C. Wong, and L. Ulsh. 1983. Shedding and immunosuppressive activity of YAC lymphoma cell gangliosides. Cancer Res. 43:3808-3813.

5. Miller, H. C., and W. J. Esselman. 1975. Modulation of the immune response by antigen-reactive lymphocytes after cultivation with gangliosides. J. Immunol. 115:839-843.

6. Lengle, E. E., R. Krishnaraj, and R. G. Kemp. 1979. Inhibition of the lectin-induced mitogenic response of thymocytes by glycolipids. Cancer Res. 39:817-822.

7. Whisler, R. L., and A. J. Yates. 1980. Regulation of lymphocyte responses by human gangliosides. J. Immunol. 125:2106-2111.

8. Ladisch, S., C. Wong, L. Ulsh, and A.-M. Delay. 1981. Suppression of antigen-specific lymphocyte blastogenic responses by tumor cell-derived gangliosides. Clin. Res. 29:528A.

9. Hersh, E. M., and J. E. Harris. 1968. Macrophage-lymphocyte interaction in the antigen-induced blastogenic response of human peripheral blood leukocytes. J. Immunol. 100:1184-1194.

10. Cline, M. J., and V. C. Sweet. 1968. The interaction of human monocytes and lymphocytes. J. Exp. Med. 128:1309-1325. 
11. Svennerholm, L. 1957. Quantitative estimation of sialic acids. II. A colorimetric resorcinol-hydrochloric acid method. Biochim. Biophys. Acta. 24:604-611.

12. Boyum, A. 1968. Isolation of mononuclear cells and granulocytes from human blood. Isolation of mononuclear cells by one centrifugation, and of granulocytes by combining centrifugation and sedimentation at 1 g. Scand. J. Clin. Lab. Invest. Suppl. 21:77-89.

13. Koski, I., D. G. Poplack, and R. M. Blaese. 1976. A nonspecific esterase stain for the identification of monocytes and macrophages. In Vitro Methods in Cell-Mediated and Tumor Immunity. B. R. Bloom and J. R. David, editors. Academic Press, Inc., New York. 359-362.

14. Ladisch, S., W. Ho, D. Matheson, R. Pilkington, and G. Hartman. 1982. Immunologic and clinical effects of repeated blood exchange in familial erythrophagocytic lymphohistiocytosis. Blood. 60:814-821.

15. Holmgren, J., H. Elwing, P. Fredman, and L. Svennerholm. 1980. Polystyrene-adsorbed gangliosides for investigation of the structure of the tetanus-toxin receptor. Eur. J. Biochem. 106:371-379.
16. Scala, G., and J. J. Oppenheim. 1983. Antigen presentation by human monocytes: evidence for stimulant processing and requirement for interleukin 1. J. Immunol. 131:1160-1166.

17. Skipski, U. P., N. Katopodis, S. S. Prendergast, and C. C. Stock. 1975. Serum of normal rats and Morris hepatoma 5123Fcbearing rats. Biochem. Biophys. Res. Commun. 67:1122-1127.

18. Lengle, E. E. 1979. Increased levels of lipid-bound sialic acid in thymic lymphocytes and plasma of leukemic AKR/J mice. J. Nat. Cancer Inst. 62:1565-1567.

19. Engers, H. D., G. D. Sorenson, G. Terres, C. Horvath, and K. T. Brunner. 1982. Functional activity in vivo of effector T cell populations. Anti-tumor activity exhibited by allogeneic mixed leukocyte culture cells. J. Immunol. 129:1292-1298.

20. Engers, H. D., A. L. Glasebrook, and G. D. Sorenson. 1982. Allogeneic tumor rejection induced by the intravenous injection of Lyt-2 ${ }^{+}$cytolytic T lymphocyte clones. J. Exp. Med. 156:1280-1285.

21. Krishnaraj, R., J. Lin, and R. G. Kemp. 1983. Lectin- and ionophore-stimulated $\mathrm{Ca}^{2+}$ influx in murine lymphocytes: inhibition by disialo-ganglioside. Cell. Immunol. 78:152-160. 